\title{
New symmetries of Black-Scholes equation
}

\author{
Tshidiso Masebe \\ Tshwane University of Technology \\ Maths,Science\& Tech Department \\ No 2 Aubrey Matlala Road, Soshanguve H \\ South Africa \\ masebetp@tut.ac.za \\ University of South Africa \\ Department of Mathematical Sciences \\ Preller street 32, City of Tshwane \\ South Africa \\ manaljm@unisa.ac.za
}

\begin{abstract}
Lie Point symmetries and Euler's formula for solving second order ordinary linear differential equationsare used to determine symmetries for the one-dimensional BlackScholes equation. One symmetry is utilized todetermine an invariant solutions
\end{abstract}

Key-Words: Lie Point Symmetries, BlackScholes equation, invariant solution.

\section{INTRODUCTION}

The past few years the world experienced an economic meltdown in part due to inappropriate management of financial securities. A derivative financial security may be defined as a security whose value depends on the value of other more basic underlying variables which may be priced or traded securities, prices of commodities or stock indices [5].

The Black-Scholes equation is a partial differential equation that governs the value of financial derivatives. Determining the value of derivatives had been a problem in finance for almost 70 years since 1990 . In the early 70s, Black and Scholes made a pioneering contribution to finance by developing a BlackScholes equation under very restrictive assumptions and the option valuation formula. Scholes obtained a Nobel Prize for economics in 1997 for his contribution (Black had passed on in 1995 and could not receive the prize personally) [5].The widely used one-dimensional model (one state variable plus time) is described by the equation

$$
u_{t}+\frac{1}{2} A^{2} x^{2} u_{x x}+B x u_{x}-C u=0
$$

Lie group theory is applied in the mathematical model of finance. In their work ([2]), Ibragimov and Gazizov determined the complete symmetry analysis of the one-dimensional Black-Scholes equation and constructed invariant solutions for some examples. In the present project we determine the same using Euler formulas.

The one-dimensional Black-Scholes equation (1) is transformed using the following change of variables.

$$
\begin{aligned}
& u_{x}=\frac{\partial u}{\partial x} \\
& x u_{x}=\frac{\partial u}{\frac{\partial x}{x}}=\frac{\partial u}{\partial \ln x}
\end{aligned}
$$

Let

$$
\begin{aligned}
& r=\ln x, \text { then } \\
& \frac{\partial r}{\partial x}=\frac{1}{x} \\
& \frac{\partial x}{\partial r}=x
\end{aligned}
$$

We therefore express

$$
x u_{x}=\frac{\partial u}{\partial r}
$$

with constant coefficients A, B and C. [2] 
Also,

$$
\begin{aligned}
& u_{x x}=\frac{\partial}{\partial x}\left(\frac{\partial u}{\partial x}\right) \\
& x^{2} u_{x x}=x^{2} \frac{\partial}{\partial x}\left(\frac{\partial u}{\partial x}\right) \\
& =x\left\{\frac{\partial}{\frac{\partial x}{x}}\left(\frac{\partial u}{\partial x}\right)\right\} \\
& =x\left\{\frac{\partial}{\partial \ln x}\left(\frac{\partial u}{\partial x}\right)\right\} \\
& =x\left\{\frac{\partial}{\partial r}\left(\frac{\partial u}{\partial x}\right)\right\} \\
& =x\left\{\frac{\partial}{\partial r}\left(\frac{1}{x} \frac{\partial u}{\partial \ln x}\right)\right\}=x\left\{\frac{\partial}{\partial r}\left(\frac{1}{x} \frac{\partial u}{\partial r}\right)\right\} \\
& =x\left\{-\frac{1}{x^{2}} \frac{\partial r}{\partial x} \frac{\partial u}{\partial r}+\frac{1}{x} \frac{\partial^{2} u}{\partial r^{2}}\right\} \\
& =x\left\{-\frac{1}{x} \frac{\partial u}{\partial r}+\frac{1}{x} \frac{\partial^{2} u}{\partial r^{2}}\right\} \\
& =-\frac{\partial u}{\partial r}+\frac{\partial^{2} u}{\partial r^{2}}
\end{aligned}
$$

Therefore

$$
\begin{aligned}
& x u_{x}=u_{r} \\
& x^{2} u_{x x}=u_{r r}-u_{r}
\end{aligned}
$$

where $r$ is given by (3). We substitute for (8) in equation (1) and define

$$
D=B-\frac{A^{2}}{2},
$$

then the Black-Scholes one dimensional equation transforms to

$$
u_{t}+\frac{1}{2} A^{2} u_{r r}+D u_{r}-C u=0 .
$$

Therefore

$$
\begin{aligned}
& x u_{x}=u_{r} \\
& x^{2} u_{x x}=u_{r r}-u_{r}
\end{aligned}
$$

where $r$ is given by (3). We substitute for (8) in equation (1) and define

$$
D=B-\frac{A^{2}}{2},
$$

then the Black-Scholes one dimensional equation transforms to

$$
u_{t}+\frac{1}{2} A^{2} u_{r r}+D u_{r}-C u=0 .
$$

\section{SOLUTION OF DETERMINING EQUATION}

The infinitesimal generator for point symmetry admitted by equation (10) is of the form

$$
X=\xi^{1}(t, r) \frac{\partial}{\partial t}+\xi^{2}(t, r) \frac{\partial}{\partial r}+\eta(t, r) \frac{\partial}{\partial u}
$$

Its first and second prolongations are given by

$$
X^{(2)}=X+\eta_{t}^{(1)} \frac{\partial}{\partial u_{t}}+\eta_{r}^{(1)} \frac{\partial}{\partial u_{r}}+\eta_{r r}^{(2)} \frac{\partial}{\partial u_{r r}}
$$

where $X$ is defined by equation (10).

The determining equation is given by

$$
\eta_{t}^{(1)}+\frac{1}{2} A^{2} \eta_{r r}^{(2)}+D \eta_{r}^{(1)}-C \eta=0
$$

when

$$
u_{r r}=\left(-\frac{2}{A^{2}}\right)\left[u_{t}+D u_{r}-C u\right]
$$

where we define the following from ([1],[3])

$$
\begin{aligned}
& \eta=f u+g \\
& \eta_{t}^{(1)}=g_{t}+f_{t} u+\left[f-\xi_{t}^{1}\right] u_{t}-\xi_{t}^{2} u_{r} \\
& \eta_{r}^{(1)}=g_{r}+f_{r} u+\left[f-\xi_{r}^{2}\right] u_{r}-\xi_{r}^{1} u_{t} \\
& \eta_{r r}^{(2)}=g_{r r}+f_{r r} u+\left[2 f_{r}-\xi_{r r}^{2}\right] u_{r}-\xi_{r r}^{1} u_{t} \\
& \quad+\left[f-2 \xi_{r}^{2}\right] u_{r r}-2 \xi_{r}^{1} u_{t r}
\end{aligned}
$$

The substitutions of $\eta_{t}^{(1)}, \eta_{r}^{(1)}$ and $\eta_{r r}^{(2)}$ in the determining equation yields that

$$
\begin{aligned}
& g_{t}+f_{t} u+\left[f-\xi_{t}^{1}\right] u_{t}-\xi_{t}^{2} u_{r}+\left(\frac{1}{2} A^{2}\right)\left\{g_{r r}+f_{r r} u\right. \\
& +\left[2 f_{r}-\xi_{r r}^{2}\right] u_{r}-\xi_{r r}^{1} u_{t}+\left[f-2 \xi_{r}^{2}\right] \\
& \left.\left(-\frac{2}{A^{2}}\left[u_{t}+D u_{r}-C u\right]\right)-2 \xi_{r}^{1} u_{t r}\right\}+(D)\left[g_{r}+f_{r} u\right. \\
& \left.+\left[f-\xi_{r}^{2}\right] u_{r}-\xi_{r}^{1} u_{t}\right]-C f u-C g=0
\end{aligned}
$$

We set the coefficients of $u_{r}, u_{t r}, u_{t}$ and those free of these variables to zero. We thus have the following defining equations

$$
\begin{aligned}
& u_{t r}: \xi_{r}^{1}=0 \\
& u_{t}: \quad-\xi_{t}^{1}+2 \xi_{r}^{2}=0 \\
& u_{r}: \quad-\xi_{t}^{2}+A^{2} f_{r}+D \xi_{r}^{2}-\frac{1}{2} A^{2} \xi_{r r}^{2}=0 \\
& u_{r}^{0} \quad: \quad g_{t}+\frac{1}{2} A^{2} g_{r r}+D g_{r}-C g=0 \\
& u \quad: \quad f_{t}+\frac{1}{2} A^{2} f_{r r}+D f_{r}-2 C \xi_{r}^{2}=0
\end{aligned}
$$


From defining equation (17) we have that

$$
\xi_{r r}^{2}=0
$$

Thus

$$
\xi^{2}=a r+b
$$

which can be expressed using Euler formula with infinitesimal $\omega$ as

$$
\begin{aligned}
& \xi^{2}=\frac{a \sin \left(\frac{\omega r}{i}\right)+b \phi \cos \left(\frac{\omega r}{i}\right)}{-i \omega} \\
& \text { where } \phi=\sin \left(\frac{\omega}{i}\right) .
\end{aligned}
$$

We differentiate equation (23) with respect to $r$ and $t$ and obtain the following equations

$$
\begin{gathered}
\xi_{r}^{2}=a \cos \left(\frac{\omega r}{i}\right)-b \phi \sin \left(\frac{\omega r}{i}\right), \\
\xi_{r r}^{2}=\frac{-\omega}{i} a \sin \left(\frac{\omega r}{i}\right)-\frac{\omega}{i} b \phi \cos \left(\frac{\omega r}{i}\right), \\
\xi_{t}^{2}=\frac{\dot{a} \sin \left(\frac{\omega r}{i}\right)+\dot{b} \phi \cos \left(\frac{\omega r}{i}\right)}{-i \omega}
\end{gathered}
$$

and from defining equation (17) we have $\xi_{t}^{1}=2 \xi_{r}^{2}$ which implies that

$$
\xi^{1}=2 a t \cos \left(\frac{\omega r}{i}\right)-2 b t \phi \sin \left(\frac{\omega r}{i}\right)+C
$$

We substitute for equations (24), (25) and (26) in the defining equation (18) to get the expression for $f_{r}$ given by

$$
\begin{aligned}
& f_{r}=\cos \left(\frac{\omega r}{i}\right)\left\{-\frac{\omega b \phi}{2 i}-\frac{\dot{b} \phi}{A^{2} i \omega}-\frac{D a}{A^{2}}\right\} \\
& +\sin \left(\frac{\omega r}{i}\right)\left\{-\frac{\omega a}{2 i}-\frac{\dot{a}}{A^{2} i \omega}+\frac{D b \phi}{A^{2}}\right\}
\end{aligned}
$$

Integrating equation (28) with respect to $r$ gives the expression for $f$

$$
\begin{aligned}
& f=\sin \left(\frac{\omega r}{i}\right)\left\{-\frac{b \phi}{2}-\frac{\dot{b} \phi}{A^{2} \omega^{2}}-\frac{D i a}{A^{2} \omega}\right\} \\
& +\cos \left(\frac{\omega r}{i}\right)\left\{\frac{a}{2}+\frac{\dot{a}}{A^{2} \omega^{2}}-\frac{D i b \phi}{A^{2} \omega}\right\}+k(t)
\end{aligned}
$$

We use the equations (28) and (29) to get the expressions for $f_{r r}$ and $f_{t}$ given by

$$
\begin{aligned}
& f_{r r}=\sin \left(\frac{\omega r}{i}\right)\left\{-\frac{\omega^{2} b \phi}{2}-\frac{\dot{b} \phi}{A^{2}}+\frac{D a \omega}{A^{2} i}\right\} \\
& +\cos \left(\frac{\omega r}{i}\right)\left\{\frac{\omega^{2} a}{2}+\frac{\dot{a}}{A^{2}}+\frac{D b \omega \phi}{A^{2} i}\right\}
\end{aligned}
$$

and

$$
\begin{aligned}
& f_{t}=\sin \left(\frac{\omega r}{i}\right)\left\{-\frac{\dot{b} \phi}{2}-\frac{\ddot{b} \phi}{A^{2} \omega^{2}}-\frac{D i \dot{a}}{A^{2} \omega}\right\} \\
& +\cos \left(\frac{\omega r}{i}\right)\left\{\frac{\dot{a}}{2}+\frac{\ddot{a}}{A^{2} \omega^{2}}-\frac{D i \dot{a} \phi}{A^{2} \omega}\right\}+k^{\prime}(t)
\end{aligned}
$$

We substitute for the equations (24), (28), (30) and (31) in the defining equation (20) and solve the equation

$$
\begin{aligned}
& \sin \left(\frac{\omega r}{i}\right)\left\{-\frac{\dot{b} \phi}{2}-\frac{\ddot{b} \phi}{A^{2} \omega^{2}}-\frac{D i \dot{a}}{A^{2} \omega}+2 C b \phi\right\} \\
& +\cos \left(\frac{\omega r}{i}\right)\left\{\frac{\dot{a}}{2}+\frac{\ddot{a}}{A^{2} \omega^{2}}-\frac{D i \dot{b} \phi}{A^{2} \omega}-2 C a\right\}+k^{\prime}(t) \\
& +\sin \left(\frac{\omega r}{i}\right)\left\{-\frac{b A^{2} \omega^{2} \phi}{4}-\frac{\dot{b} \phi}{2}+\frac{D a \omega}{2 i}\right\}+ \\
& \cos \left(\frac{\omega r}{i}\right)\left\{\frac{a A^{2} \omega^{2}}{4}+\frac{\dot{a}}{2}+\frac{D b \omega \phi}{2 i}\right\}+ \\
& \cos \left(\frac{\omega r}{i}\right)\left\{-\frac{D b \omega \phi}{2 i}-\frac{D \dot{b} \phi}{A^{2} i \omega}-\frac{D^{2} a}{A^{2}}\right\}+ \\
& \sin \left(\frac{\omega r}{i}\right)\left\{-\frac{D a \omega}{2 i}-\frac{D \dot{a}}{A^{2} i \omega}+\frac{D^{2} b \phi}{A^{2}}\right\}=0
\end{aligned}
$$

We collect all the coefficients of sine function together and equate them to zero. Similarly with the cosine function. For the coefficients of sine function we have:

$$
\begin{aligned}
& -\frac{\dot{b} \phi}{2}-\frac{\ddot{b} \phi}{A^{2} \omega^{2}}-\frac{D i \dot{a}}{A^{2} \omega}-\frac{b A^{2} \omega^{2} \phi}{4}-\frac{\dot{b} \phi}{2} \\
& -\frac{D a \omega}{2 i}+\frac{D a \omega}{2 i}-\frac{D \dot{a}}{A^{2} \omega i}-\frac{D^{2} b \phi}{A^{2}}+2 C b \phi=0
\end{aligned}
$$

which simplifies to a second-order ordinary linear differential equation

$$
\ddot{b}+\dot{b} A^{2} \omega^{2}+\frac{b A^{4} \omega^{4}}{4}-\frac{D^{2} b}{A^{2}}+2 C A^{2} \omega^{2} b=0
$$

Solving for equation (34) we proceed as follows. Let

$$
\begin{aligned}
& \beta=\frac{A^{2} \omega^{2}}{2}, \quad \text { and } \\
& k_{1}=-\frac{D^{2}}{A^{2}}-2 C
\end{aligned}
$$

We also set

$$
\begin{aligned}
& \alpha_{1}=b \beta^{2}-k, \text { then } \\
& \dot{\alpha_{1}}=\dot{b} \beta^{2}, \\
& \ddot{\alpha}_{1}=\ddot{b} \beta^{2}
\end{aligned}
$$


Equation (34) transforms to

$$
\ddot{\alpha_{1}}+2 \dot{\alpha}_{1}+\alpha_{1} \beta^{2}=0 .
$$

To find the solution of equation (37) we proceed as follows. We set

$$
\alpha_{1}=c z
$$

where $c=c(t), z=z(t)$. Then

$$
\begin{aligned}
& \alpha_{1}^{\prime}=c^{\prime} z+c z^{\prime} \\
& \alpha_{1}^{\prime \prime}=c^{\prime \prime} z+2 c^{\prime} z^{\prime}+c z^{\prime \prime}
\end{aligned}
$$

We substitute for equations (38), (39) and (40) in equation (37) and after rearranging we solve the equation

$$
c z^{\prime \prime}+\left(2 c+2 c^{\prime}\right) z+\left(c^{\prime \prime}+2 c^{\prime}+\beta^{2} c\right) z=0
$$

The choice for $c$ is such that

$$
2 c+2 c^{\prime}=0
$$

whence

$$
c=e^{-t} \text {. }
$$

The equation (41) simplifies to

$$
z^{\prime \prime}+\left(\beta^{2}-1\right) z=0
$$

The solution for (37) is now written

$$
\begin{aligned}
& \alpha_{1}=e^{-t}\left(C_{1} \frac{\sin \bar{\omega} \cos \bar{\omega} t}{\bar{\omega}}\right) \\
& +C_{2} e^{-t} \frac{\sin \bar{\omega} t}{\bar{\omega}}
\end{aligned}
$$

so that when $\beta= \pm 1$ or $\omega \rightarrow 0$ the solution for $z$ is linear, and we define

$$
\bar{\omega}=\sqrt{\beta^{2}-1}
$$

We substitute for $b$ in equation (36) to obtain that

$$
\begin{aligned}
& b=\frac{e^{-t}}{\beta^{2}}\left\{\left(C_{1} \frac{\sin \bar{\omega} \cos \bar{\omega} t}{\bar{\omega}}\right)\right. \\
& \left.+C_{2} e^{-t} \frac{\sin \bar{\omega} t}{\bar{\omega}}\right\}+\frac{D^{2}}{\beta^{2} A^{2}}+\frac{4 C}{\beta}
\end{aligned}
$$

Similarly for the coefficients of the cosine function we have

$$
\begin{aligned}
& \frac{\dot{a}}{2}+\frac{\ddot{a} \phi}{A^{2} \omega^{2}}-\frac{D i \dot{b} \phi}{A^{2} \omega}+\frac{a A^{2} \omega^{2} \phi}{4}+\frac{\dot{a}}{2} \\
& +\frac{D b \omega \phi}{2 i}-\frac{D b \omega \phi}{2 i}-\frac{D \dot{b} \phi}{A^{2} \omega i}-\frac{D^{2} a}{A^{2}}+2 a C=0
\end{aligned}
$$

which simplifies to a second-order ordinary linear differential equation

$$
\ddot{a}+\dot{a} A^{2} \omega^{2}+\frac{a A^{4} \omega^{4}}{4}-\frac{a D^{2}}{A^{2}}-2 a A^{2} \omega^{2} C=0
$$

Solving for equation(49) we find the solution for $a$ to be

$$
\begin{aligned}
& a=\frac{e^{-t}}{\beta^{2}}\left\{\left(C_{3} \frac{\sin \bar{\omega} \cos \bar{\omega} t}{\bar{\omega}}\right)+C_{4} \frac{\sin \bar{\omega} t}{\bar{\omega}}\right\} \\
& +\frac{D^{2}}{\beta^{2} A^{2}}-\frac{4 C}{\beta}
\end{aligned}
$$

and we also have that

$$
k^{\prime}(t)=0 \quad \Rightarrow \quad k(t)=C_{5}
$$

We differentiate equations (47) and (50) to obtain expressions for $\dot{a}$ and $\dot{b}$

$$
\begin{aligned}
\dot{a} & =-\frac{e^{-t}}{\beta^{2}}\left(C_{3} \frac{\sin \bar{\omega} \cos \bar{\omega} t}{\bar{\omega}}\right. \\
& \left.+C_{4} \frac{\sin \bar{\omega} t}{\bar{\omega}}\right)+\frac{e^{-t}}{\beta^{2}}\left(-C_{3} \sin \bar{\omega} \sin \bar{\omega} t+C_{4} \cos \bar{\omega} t\right)
\end{aligned}
$$

\section{Similarly}

$$
\begin{array}{r}
\dot{b}=-\frac{e^{-t}}{\beta^{2}}\left(C_{1} \frac{\sin \bar{\omega} \cos \bar{\omega} t}{\bar{\omega}}+C_{2} \frac{\sin \bar{\omega} t}{\bar{\omega}}\right) \\
+\frac{e^{-t}}{\beta^{2}}\left(-C_{1} \sin \bar{\omega} \sin \bar{\omega} t+C_{2} \cos \bar{\omega} t\right)
\end{array}
$$

We substitute for equations (35),(47),(50),(51),(52) and (53) in equation (29) and get the expression for 
$f$ given as

$$
\begin{aligned}
& f=\sin \left(\frac{\omega r}{i}\right)\left\{-\frac{C_{1} \phi e^{-t} \sin \bar{\omega} \cos \bar{\omega} t}{2 \beta^{2} \bar{\omega}}\right. \\
& -\frac{C_{2} \phi e^{-t} \sin \bar{\omega} t}{2 \beta^{2} \bar{\omega}}-\frac{D^{2} \phi}{2 \beta^{3} A^{2}}-\frac{4 C \phi}{\beta}+\frac{C_{1} \phi e^{-t} \sin \bar{\omega} \cos \bar{\omega} t}{2 \beta^{3} \bar{\omega}} \\
& +\frac{C_{2} \phi e^{-t} \sin \bar{\omega} t}{2 \beta^{3} \bar{\omega}}+\frac{C_{1} \phi e^{-t} \sin \bar{\omega} \cos \bar{\omega} t}{2 \beta^{3}} \\
& -\frac{C_{2} \phi e^{-t} \cos \bar{\omega} t}{2 \beta^{3}}-\frac{C_{3} D i \omega e^{-t} \sin \bar{\omega} \cos \bar{\omega} t}{2 \beta^{3}} \\
& \left.-\frac{C_{4} D i \omega e^{-t} \sin \bar{\omega} t}{2 \beta^{3}}-\frac{D^{3} i \omega}{2 \beta^{3} A^{2}}\right\} \\
& +\cos \left(\frac{\omega r}{i}\right)\left\{\frac{C_{3} e^{-t} \sin \bar{\omega} \cos \bar{\omega} t}{2 \beta^{2} \bar{\omega}}+\frac{C_{4} e^{-t} \sin \bar{\omega} t}{2 \beta^{2} \bar{\omega}}\right. \\
& +\frac{D^{2}}{2 \beta^{3} A^{2}}-\frac{4 C \phi}{\beta}-\frac{C_{3} e^{-t} \sin \bar{\omega} \cos \bar{\omega} t}{2 \beta^{3} \bar{\omega}}-\frac{C_{4} e^{-t} \sin \bar{\omega} t}{2 \beta^{3} \bar{\omega}} \\
& -\frac{C_{3} e^{-t} \sin \bar{\omega} \sin \bar{\omega} t}{2 \beta^{3}} \\
& -\frac{C_{4} e^{-t} \cos \bar{\omega} t}{2 \beta^{3}}-\frac{C_{1} \phi D i \omega e^{-t} \sin \bar{\omega} \cos \bar{\omega} t}{2 \beta^{3}} \\
& \left.-\frac{C_{2} \phi D i \omega e^{-t} \sin \bar{\omega} t}{2 \beta^{3}}-\frac{D^{3} \phi i \omega}{2 \beta^{3} A^{2}}\right\}+C_{5}
\end{aligned}
$$

\section{A. Infinitesimals}

The linearly independent solutions of the defining equations (15) lead to the infinitesimals

$$
\begin{aligned}
& \xi^{1}=\cos \left(\frac{\omega r}{i}\right)\left\{\frac { 2 t e ^ { - t } } { \beta ^ { 2 } \overline { \omega } } \left\{\left(C_{3} \sin \bar{\omega} \cos \bar{\omega} t\right)\right.\right. \\
& \left.\left.+C_{4} \sin \bar{\omega} t\right\}+\frac{2 t D^{2}}{\beta^{2} A^{2}}\right\} \\
& -\sin \left(\frac{\omega r}{i}\right)\left\{\frac{2 t \phi e^{-t}}{\beta^{2} \bar{\omega}}\right. \\
& \left\{\left(C_{1} \sin \bar{\omega} \cos \bar{\omega} t\right)+C_{2} \sin \bar{\omega} t\right\} \\
& \left.+\frac{2 t \phi D^{2}}{\beta^{2} A^{2}}+C_{6}\right\} \\
& \xi^{2}=\sin \left(\frac{\omega r}{i}\right)\left\{\frac { e ^ { - t } } { - \beta ^ { 2 } \omega \overline { \omega } i } \left(C_{3} \sin \bar{\omega} \cos \bar{\omega} t\right.\right. \\
& \left.\left.+C_{4} \sin \bar{\omega} t\right)-\frac{D^{2}}{i \omega \beta^{2} A^{2}}\right\} \\
& +\cos \left(\frac{\omega r}{i}\right)\left\{\frac { e ^ { - t } \phi } { - \beta ^ { 2 } \omega \overline { \omega } i } \left(C_{1} \sin \bar{\omega} \cos \bar{\omega} t\right.\right. \\
& \left.\left.-C_{2} \sin \bar{\omega} t\right)-\frac{D^{2} \phi}{i \omega \beta^{2} A^{2}}\right\}
\end{aligned}
$$

$$
\begin{aligned}
& f=\sin \left(\frac{\omega r}{i}\right)\left\{-\frac{C_{1} \phi e^{-t} \sin \bar{\omega} \cos \bar{\omega} t}{2 \beta^{2} \bar{\omega}}\right. \\
& -\frac{C_{2} \phi e^{-t} \sin \bar{\omega} t}{2 \beta^{2} \bar{\omega}}-\frac{D^{2} \phi}{2 \beta^{3} A^{2}} \\
& -\frac{4 C \phi}{\beta}+\frac{C_{1} \phi e^{-t} \sin \bar{\omega} \cos \bar{\omega} t}{2 \beta^{3} \bar{\omega}} \\
& +\frac{C_{2} \phi e^{-t} \sin \bar{\omega} t}{2 \beta^{3} \bar{\omega}}+\frac{C_{1} \phi e^{-t} \sin \bar{\omega} \cos \bar{\omega} t}{2 \beta^{3}} \\
& -\frac{C_{2} \phi e^{-t} \cos \bar{\omega} t}{2 \beta^{3}}-\frac{C_{3} D i \omega e^{-t} \sin \bar{\omega} \cos \bar{\omega} t}{2 \beta^{3}} \\
& \left.-\frac{C_{4} D i \omega e^{-t} \sin \bar{\omega} t}{2 \beta^{3}}-\frac{D^{3} i \omega}{2 \beta^{3} A^{2}}\right\} \\
& +\cos \left(\frac{\omega r}{i}\right)\left\{\frac{C_{3} e^{-t} \sin \bar{\omega} \cos \bar{\omega} t}{2 \beta^{2} \bar{\omega}}+\frac{C_{4} e^{-t} \sin \bar{\omega} t}{2 \beta^{2} \bar{\omega}}\right. \\
& +\frac{D^{2}}{2 \beta^{3} A^{2}}-\frac{4 C \phi}{\beta}-\frac{C_{3} e^{-t} \sin \bar{\omega} \cos \bar{\omega} t}{2 \beta^{3} \bar{\omega}} \\
& -\frac{C_{4} e^{-t} \sin \bar{\omega} t}{2 \beta^{3} \bar{\omega}}-\frac{C_{3} e^{-t} \sin \bar{\omega} \sin \bar{\omega} t}{2 \beta^{3}} \\
& -\frac{C_{4} e^{-t} \cos \bar{\omega} t}{2 \beta^{3}}-\frac{C_{1} \phi D i \omega e^{-t} \sin \bar{\omega} \cos \bar{\omega} t}{2 \beta^{3}} \\
& \left.-\frac{C_{2} \phi D i \omega e^{-t} \sin \bar{\omega} t}{2 \beta^{3}}-\frac{D^{3} \phi i \omega}{2 \beta^{3} A^{2}}\right\}+C_{5}
\end{aligned}
$$

\section{B. The symmetries}

According to (15), the infinitesimals: (57), (55) and (56), lead to the generators

$$
\begin{aligned}
& X_{1}=\left(-\frac{2 t e^{-t} \phi}{\beta^{2} \bar{\omega}} \sin \bar{\omega} \cos \bar{\omega} t \sin \left(\frac{\omega r}{i}\right)\right) \frac{\partial}{\partial t} \\
& +\left(\frac{e^{-t} i \phi}{\beta^{2} \bar{\omega} \omega} \sin \bar{\omega} \cos \bar{\omega} t \cos \left(\frac{\omega r}{i}\right)\right) \frac{\partial}{\partial r} \\
& +\left\{-\frac{e^{-t} \phi}{2 \beta^{2} \bar{\omega}} \sin \bar{\omega} \cos \bar{\omega} t \sin \left(\frac{\omega r}{i}\right)\right. \\
& +\frac{e^{-t} \phi}{2 \beta^{3} \bar{\omega}} \sin \bar{\omega} \cos \bar{\omega} t \sin \left(\frac{\omega r}{i}\right) \\
& +\frac{e^{-t} \phi}{2 \beta^{3}} \sin \bar{\omega} \cos \bar{\omega} t \sin \left(\frac{\omega r}{i}\right) \\
& \left.-\frac{D i \phi \omega e^{-t} \sin \bar{\omega} \cos \bar{\omega} t}{2 \beta^{3}} \cos \left(\frac{\omega r}{i}\right)\right\} u \frac{\partial}{\partial u}
\end{aligned}
$$




$$
\begin{aligned}
& X_{2}=\left(-\frac{2 t e^{-t} \phi}{\beta^{2} \bar{\omega}} \sin \bar{\omega} t \sin \left(\frac{\omega r}{i}\right)\right) \frac{\partial}{\partial t} \\
& -\left(\frac{e^{-t} i \phi}{\beta^{2} \bar{\omega} \omega} \sin \bar{\omega} t \cos \left(\frac{\omega r}{i}\right)\right) \frac{\partial}{\partial r} \\
& +\left\{-\frac{e^{-t} \phi}{2 \beta^{2} \bar{\omega}} \sin \bar{\omega} t \sin \left(\frac{\omega r}{i}\right)\right. \\
& +\frac{e^{-t} \phi}{2 \beta^{3} \bar{\omega}} \sin \bar{\omega} t \sin \left(\frac{\omega r}{i}\right) \\
& -\frac{e^{-t} \phi}{2 \beta^{3}} \cos \bar{\omega} t \sin \left(\frac{\omega r}{i}\right) \\
& \left.-\frac{D i \phi \omega e^{-t} \sin \bar{\omega} t}{2 \beta^{3}} \cos \left(\frac{\omega r}{i}\right)\right\} u \frac{\partial}{\partial u}
\end{aligned}
$$$$
X_{3}=\left(\frac{2 t e^{-t}}{\beta^{2} \bar{\omega}} \sin \bar{\omega} \cos \bar{\omega} t \cos \left(\frac{\omega r}{i}\right)\right) \frac{\partial}{\partial t}
$$$$
+\left(\frac{e^{-t} i}{\beta^{2} \bar{\omega} \omega} \sin \bar{\omega} \cos \bar{\omega} t \cos \left(\frac{\omega r}{i}\right)\right) \frac{\partial}{\partial r}
$$$$
+\left\{\frac{e^{-t}}{2 \beta^{2} \bar{\omega}} \sin \bar{\omega} \cos \bar{\omega} t \cos \left(\frac{\omega r}{i}\right)\right.
$$$$
-\frac{e^{-t}}{2 \beta^{3} \bar{\omega}} \sin \bar{\omega} \cos \bar{\omega} t \cos \left(\frac{\omega r}{i}\right)
$$$$
-\frac{e^{-t} \phi}{2 \beta^{3}} \sin \bar{\omega} \sin \bar{\omega} t \cos \left(\frac{\omega r}{i}\right)
$$$$
\left.-\frac{D i \omega e^{-t} \sin \bar{\omega} \cos \bar{\omega} t}{2 \beta^{3}} \sin \left(\frac{\omega r}{i}\right)\right\} u \frac{\partial}{\partial u}
$$$$
X_{4}=\left(\frac{2 t e^{-t} \phi}{\beta^{2} \bar{\omega}} \sin \bar{\omega} t \cos \left(\frac{\omega r}{i}\right)\right) \frac{\partial}{\partial t}
$$$$
+\left(\frac{e^{-t} i \phi}{\beta^{2} \bar{\omega} \omega} \sin \bar{\omega} t \sin \left(\frac{\omega r}{i}\right)\right) \frac{\partial}{\partial r}
$$$$
+\left\{\frac{e^{-t}}{2 \beta^{2} \bar{\omega}} \sin \bar{\omega} t \cos \left(\frac{\omega r}{i}\right)\right.
$$$$
-\frac{e^{-t}}{2 \beta^{3} \bar{\omega}} \sin \bar{\omega} t \cos \left(\frac{\omega r}{i}\right)
$$$$
+\frac{e^{-t}}{2 \beta^{3}} \cos \bar{\omega} t \cos \left(\frac{\omega r}{i}\right)
$$$$
\left.-\frac{D i \omega e^{-t} \sin \bar{\omega} t}{2 \beta^{3}} \sin \left(\frac{\omega r}{i}\right)\right\} u \frac{\partial}{\partial u}
$$$$
X_{5}=\frac{2 t D^{2}}{\beta^{2} A^{2}}\left(\cos \left(\frac{\omega r}{i}\right)-\phi \sin \left(\frac{\omega r}{i}\right)\right) \frac{\partial}{\partial t}
$$$$
+\frac{D^{2}}{\omega \beta^{2} A^{2}}\left(\phi \cos \left(\frac{\omega r}{i}\right)+\sin \left(\frac{\omega r}{i}\right)\right) \frac{\partial}{\partial r}
$$$$
-\frac{D^{2} i \omega}{2 \beta^{3} A^{2}}\left(\sin \left(\frac{\omega r}{i}\right)+\phi \cos \left(\frac{\omega r}{i}\right)\right) u \frac{\partial}{\partial u}
$$$$
X_{6}=u \frac{\partial}{\partial u}
$$

$$
\begin{gathered}
X_{7}=\frac{\partial}{\partial t} \\
X_{8}=\frac{4 C}{\beta}\left(\phi \sin \left(\frac{\omega r}{i}\right)-\cos \left(\frac{\omega r}{i}\right)\right) u \frac{\partial}{\partial u}
\end{gathered}
$$

The defining equation (19) gives an infinite symmetry

$$
X_{\infty}=g(t, r) \frac{\partial}{\partial u}
$$

\section{Invariant solutions through the symme-try $X_{3}$}

The invariants are determined from solving the equation

$$
\begin{aligned}
& X_{3} I=\left(\frac{2 t e^{-t}}{\beta^{2} \bar{\omega}} \sin \bar{\omega} \cos \bar{\omega} t \cos \left(\frac{\omega r}{i}\right)\right) \frac{\partial I}{\partial t} \\
& +\left(\frac{e^{-t} i}{\beta^{2} \bar{\omega} \omega} \sin \bar{\omega} \cos \bar{\omega} t \cos \left(\frac{\omega r}{i}\right)\right) \frac{\partial I}{\partial r} \\
& +\left\{\frac{e^{-t}}{2 \beta^{2} \bar{\omega}} \sin \bar{\omega} \cos \bar{\omega} t \cos \left(\frac{\omega r}{i}\right)\right. \\
& -\frac{e^{-t}}{2 \beta^{3} \bar{\omega}} \sin \bar{\omega} \cos \bar{\omega} t \cos \left(\frac{\omega r}{i}\right) \\
& -\frac{e^{-t} \phi}{2 \beta^{3}} \sin \bar{\omega} \sin \bar{\omega} t \cos \left(\frac{\omega r}{i}\right) \\
& \left.-\frac{D i \omega e^{-t} \sin \bar{\omega} \cos \bar{\omega} t}{2 \beta^{3}} \sin \left(\frac{\omega r}{i}\right)\right\} u \frac{\partial I}{\partial u}=0
\end{aligned}
$$

The characteristic equation of (67) is given by

$$
\begin{aligned}
& \frac{2 t e^{-t} \sin \bar{\omega} \cos \bar{\omega} t \cos \left(\frac{\omega r}{i}\right)}{\beta^{2} \bar{\omega}} \\
& =\frac{d r}{\frac{e^{-t} i \sin \bar{\omega} \sin \bar{\omega} t \cos \left(\frac{\omega r}{i}\right)}{\beta^{2} \bar{\omega} \omega}} \\
& =\frac{d u}{u e^{-t}\left\{\frac{1}{\sin \bar{\omega} \cos \bar{\omega} t \cos \left(\frac{\omega r}{i}\right)} 2 \beta^{2} \bar{\omega}\right.} \\
& -\frac{1}{\sin \bar{\omega} \cos \bar{\omega} t \cos \left(\frac{\omega r}{i}\right)} 2 \beta^{3} \bar{\omega} \\
& -\frac{1}{\sin \bar{\omega} \sin \bar{\omega} t \cos \left(\frac{\omega r}{i}\right)} 2 \beta^{3} \\
& \left.-\frac{1}{D i \omega \sin \bar{\omega} \cos \bar{\omega} t \sin \left(\frac{\omega r}{i}\right)} 2 \beta^{3}\right\}
\end{aligned}
$$


From equation (68) we have that

$$
\begin{aligned}
& \frac{d t}{\frac{2 t e^{-t} \sin \bar{\omega} \cos \bar{\omega} t \cos \left(\frac{\omega r}{i}\right)}{\beta^{2} \bar{\omega}}} \\
& =\frac{d r}{\frac{e^{-t} i \sin \bar{\omega} \sin \bar{\omega} t \cos \left(\frac{\omega r}{i}\right)}{\beta^{2} \bar{\omega} \omega}}
\end{aligned}
$$

simplifies to

$$
\frac{d t}{t}=2 \frac{\omega}{i} d r
$$

whose solution is

$$
t=C e^{\frac{2 \omega r}{i}}
$$

The first invariant is given by

$$
\psi_{1}=\frac{e^{\frac{2 \omega r}{i}}}{t}
$$

From equation (68) we also have that

$$
\begin{aligned}
& \frac{\frac{d r}{e^{-t} i \sin \bar{\omega} \sin \bar{\omega} t \cos \left(\frac{\omega r}{i}\right)}}{\beta^{2} \bar{\omega} \omega} \\
& =\frac{d u}{u e^{-t}}\left\{\frac{2 \beta^{2} \bar{\omega}}{\sin \bar{\omega} \cos \bar{\omega} t \cos \left(\frac{\omega r}{i}\right)}\right. \\
& -\frac{2 \beta^{3} \bar{\omega}}{\sin \bar{\omega} \cos \bar{\omega} t \cos \left(\frac{\omega r}{i}\right)} \\
& -\frac{1}{\sin \bar{\omega} \sin \bar{\omega} t \cos \left(\frac{\omega r}{i}\right)} 2 \beta^{3} \\
& \left.-\frac{2 \beta^{3}}{D i \omega \sin \bar{\omega} \cos \bar{\omega} t \sin \left(\frac{\omega r}{i}\right)}\right\}
\end{aligned}
$$

Equation (73) simplifies to

$$
\left(\frac{\beta-1-\bar{\omega}-D i \omega \bar{\omega} \tan \left(\frac{\omega}{i}\right)}{2 \beta}\right)\left(\frac{\omega}{i}\right) d r=\frac{d u}{u}
$$

We integrate equation (74) and obtain

$$
\begin{aligned}
& \frac{\beta \omega r}{2 \beta i}-\frac{\omega r}{2 \beta i}-\frac{\bar{\omega} \omega r}{2 \beta i} \\
& -\frac{D \bar{\omega} \omega \ln \left|\cos \left(\frac{\omega r}{i}\right)\right|}{2 \beta}+C=\ln u
\end{aligned}
$$

We approximate

$$
\begin{aligned}
& \frac{\beta \omega r}{2 \beta i}-\frac{\omega r}{2 \beta i}-\frac{\bar{\omega} \omega r}{2 \beta i} \\
& -\frac{D \bar{\omega} \omega \ln \left|\cos \left(\frac{\omega r}{i}\right)\right|}{2 \beta} \approx \frac{\omega r}{i}
\end{aligned}
$$

Integrating equation (73) we obtain

$$
\frac{u}{e^{\frac{\omega r}{i}}}=C
$$

The equation (77) simplifies to

$$
\frac{u}{e^{\frac{\omega r}{i}}}=\psi_{2}
$$

which is our second invariant. If we define

$$
\psi_{2}=h\left(\psi_{1}\right)
$$

where $\psi_{1}$ is given by equation (72), then an invariant solution is given by

$$
u=e^{\frac{\omega r}{i}} h\left(\psi_{1}\right)
$$

We differentiate equation (80) with respect to $t$ and twice with respect to $r$ and get the following expressions for $u_{t}, u_{r}$ and $u_{r r}$.

$$
\begin{gathered}
u_{t}=-\frac{2 \omega r}{i} \frac{e^{\frac{3 \omega r}{i}}}{t^{2}} h^{\prime}\left(\psi_{1}\right) \\
u_{r}=\frac{\omega}{i} e^{\frac{\omega r}{i}} h\left(\psi_{1}\right)+\frac{2 \omega}{i t} e^{\frac{3 \omega r}{i}} h^{\prime}\left(\psi_{1}\right) \\
u_{r r}=-\omega^{2} e^{\frac{\omega r}{i}} h\left(\psi_{1}\right)-\frac{2 \omega^{2}}{t} e^{\frac{3 \omega r}{i}} h^{\prime}\left(\psi_{1}\right) \\
-\frac{6 \omega^{2}}{t} e^{\frac{3 \omega r}{i}} h^{\prime}\left(\psi_{1}\right)--\frac{4 \omega^{2}}{t^{2}} e^{\frac{5 \omega r}{i}} h^{\prime \prime}\left(\psi_{1}\right)
\end{gathered}
$$

We substitute for equations (81), (82) and (83) in the original equation (9) and get the following equation

$$
\begin{aligned}
& -\frac{2 \omega r}{i} \frac{e^{\frac{3 \omega r}{i}}}{t^{2}} h^{\prime}\left(\psi_{1}\right)-\omega^{2} e^{\frac{\omega r}{i}} h\left(\psi_{1}\right) \\
& -\frac{A^{2} \omega^{2}}{t} e^{\frac{3 \omega r}{i}} h^{\prime}\left(\psi_{1}\right)-\frac{3 A^{2} \omega^{2}}{t} e^{\frac{3 \omega r}{i}} h^{\prime}\left(\psi_{1}\right) \\
& -\frac{2 A^{2} \omega^{2}}{t^{2}} e^{\frac{5 \omega r}{i}} h^{\prime \prime}\left(\psi_{1}\right)+ \\
& \frac{D \omega}{i} e^{\frac{\omega r}{i}} h\left(\psi_{1}\right)+\frac{2 D \omega}{i t} e^{\frac{3 \omega r}{i}} h^{\prime}\left(\psi_{1}\right)-C e^{\frac{\omega r}{i}} h\left(\psi_{1}\right)=0
\end{aligned}
$$

Equation (84) is a second order equation in $h\left(\psi_{1}\right)$. We rearrange it in the order of derivatives of $h\left(\psi_{1}\right)$, and apply equation (35)

$$
\begin{aligned}
& -\frac{4 \beta}{t^{2}} e^{\frac{5 \omega r}{i}} h^{\prime \prime}\left(\psi_{1}\right)-h^{\prime}\left(\psi_{1}\right)\left\{\frac{2 \omega r}{i} \frac{e^{\frac{3 \omega r}{i}}}{t^{2}}\right. \\
& +\frac{4 \beta}{t} e^{\frac{3 \omega r}{i}}+\frac{6 \beta}{t} e^{\frac{3 \omega r}{i}} \\
& \left.-\frac{2 D \omega}{i} e^{\frac{3) \omega r}{i}}\right\}+h\left(\psi_{1}\right)\left\{\frac{D k \omega}{i t} e^{\frac{\omega r}{i}}\right. \\
& \left.-\beta e^{\frac{\omega r}{i}}-C e^{\frac{\omega r}{i}}\right\}=0
\end{aligned}
$$


Letting $\omega \rightarrow 0$ equation (85) simplifies to

$-\frac{4 \beta}{t^{2}} h^{\prime \prime}\left(\psi_{1}\right)-\frac{4 \beta}{t} h^{\prime}\left(\psi_{1}\right)-\frac{6 \beta}{t} h^{\prime}\left(\psi_{1}\right)-\beta h\left(\psi_{1}\right)-C h\left(\psi_{1}\right)=0$

which can be simplified to

$$
\frac{4 \beta h^{\prime \prime}\left(\psi_{1}\right)}{t^{2}}+\frac{10 \beta h^{\prime}\left(\psi_{1}\right)}{t}+(\beta+C) h\left(\psi_{1}\right)=0 .
$$

We eliminate $t$ from equation (87) by applying the following change of variables. From equation (72) we let

$$
\begin{aligned}
& d \lambda=t d \psi_{1}, \\
& h\left(\psi_{1}\right)=h
\end{aligned}
$$

then

$$
h^{\prime}\left(\psi_{1}\right)=t h_{\lambda}
$$

and

$$
\begin{gathered}
h^{\prime \prime}\left(\psi_{1}\right)=\frac{d}{d \psi_{1}}\left\{t h_{\lambda}\right\} \\
=\frac{d t}{d \psi_{1}} h_{\lambda}+t \frac{d h_{\lambda}}{d \psi_{1}} \\
=\frac{d t}{d \psi_{1}} h_{\lambda}+t \frac{d h_{\lambda}}{\frac{1}{t} d \lambda} \\
=t^{2}\left\{h_{\lambda \lambda}-h_{\lambda} e^{-\frac{2 \omega r}{i}}\right\}
\end{gathered}
$$

For $\omega \rightarrow 0$ equation (90) simplifies to

$$
h^{\prime \prime}\left(\psi_{1}\right)=t^{2}\left\{h_{\lambda \lambda}-h_{\lambda}\right\}
$$

The substitution of equations (89), (91) transforms equation (87) to

$$
4 \beta h_{\lambda \lambda}+6 \beta h_{\lambda}+(\beta+C) h=0 .
$$

The solution to equation (92) is given by

$$
\begin{aligned}
& h=C_{1} e^{\left(\frac{-3 \beta-\sqrt{9 \beta^{2}-4 \beta(\beta+C)}}{4 \beta}\right)(\lambda)} \\
& +C_{2} e^{\left(\frac{-3 \beta+\sqrt{9 \beta^{2}-4 \beta(\beta+C)}}{4 \beta}\right)(\lambda)}
\end{aligned}
$$

Thus the invariant solution is

$$
\begin{aligned}
& u=e^{\frac{\omega r}{i}}\left\{C_{1} e^{\left(\frac{-3 \beta-\sqrt{9 \beta^{2}-4 \beta(\beta+C)}}{4 \beta}\right)(\lambda)}\right. \\
& \left.+C_{2} e^{\left(\frac{-3 \beta+\sqrt{9 \beta^{2}-4 \beta(\beta+C)}}{4 \beta}\right)(\lambda)}\right\}
\end{aligned}
$$

However the equation (94) can be expressed as

$$
\begin{aligned}
& u=e^{\frac{\omega r}{i}}\left\{C_{1} e^{\left(\frac{-3 \beta-i \sqrt{-\left(9 \beta^{2}-4 \beta(\beta+C)\right)}}{4 \beta}\right)(\lambda)}\right. \\
& \left.+C_{2} e^{\left(\frac{-3 \beta+i \sqrt{-\left(9 \beta^{2}-4 \beta(\beta+C)\right)}}{4 \beta}\right)(\lambda)}\right\}
\end{aligned}
$$

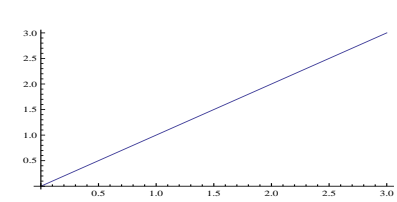

Figure 1: Plot of the solution in (101) .

This simplifies to

$$
\begin{aligned}
& u=e^{\frac{\omega r}{i}}\left\{C_{1} e^{\frac{-3}{4} \lambda} e^{-i \Delta \lambda}+C_{2} e^{\frac{-3}{4} \lambda} e^{i \Delta \lambda}\right\} \\
& \text { where } \Delta=\frac{\sqrt{-\left(9 \beta^{2}-4 \beta(\beta+C)\right)}}{4 \beta}
\end{aligned}
$$

Since $\Delta<0$, we express equation (96) as

$$
\begin{aligned}
& u=e^{\frac{\omega r}{i}}\left\{C_{1} e^{\frac{-3}{4} \lambda} \sin (\Delta \lambda)\right. \\
& \left.+C_{2} e^{\frac{-3}{4} \lambda} \cos (\Delta \lambda)\right\}
\end{aligned}
$$

We however advance the same reason that for equation (97) to return to the linear form as $\Delta \rightarrow 0$ it has to be transformed to be

$$
\begin{aligned}
& u=e^{\frac{\omega r}{i}}\left\{C_{1} e^{\frac{-3}{4} \lambda} \frac{\sin (\Delta \lambda)}{-i \Delta}+C_{2} e^{\frac{-3}{4} \lambda} \phi \frac{\cos (\Delta \lambda)}{-i \Delta}\right\} \\
& \text { where } \phi=\sin \left(\frac{\Delta}{i}\right)
\end{aligned}
$$

\section{Solutions for equation 98}

This equation (98) has some few solutions as $\omega \rightarrow 0$. We recall that

$$
\begin{aligned}
\lambda & =t \int_{\psi_{1}}^{\psi_{2}} d \frac{e^{\frac{2 \omega r}{i}}}{t} \\
& =e^{\frac{2 \omega r}{i}} \int_{\psi_{1}}^{\psi_{2}} \frac{d \frac{1}{t}}{\frac{1}{t}} \\
& =-C_{0} e^{\frac{2 \omega r}{i}} \ln t
\end{aligned}
$$

\subsection{Solution 1}

$$
\begin{gathered}
u=A e^{\frac{\omega r}{i}} e^{-\frac{3}{4} \lambda \frac{\sin (\Delta \lambda)}{-i \Delta}} \\
=A e^{\frac{\omega r}{i}} e^{\left(\frac{3}{4} C_{0} e^{\frac{2 \omega r}{i}} \ln t\right) \frac{\sin \left(\Delta\left(-C_{0} e^{\frac{2 \omega r}{i}} \ln t\right)\right)}{-i \Delta}}
\end{gathered}
$$

as $\omega \rightarrow 0$, the solution becomes

$$
u=\frac{3}{4} C_{0} t
$$

One of the assumptions of the Black-Scholes model is that the option value is perfectly linear. The 


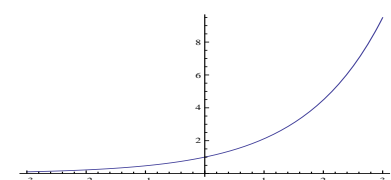

Figure 2: Plot of the solution in (103) .

linearity of the graph illustrates an important feature of the Black-Scholes model in that it provides an excellent approximation to the value of the option with variable volatility as long as mathematical expectation of the volatility is known ([4])

\subsection{Solution 2}

$$
\begin{gathered}
u=A e^{\frac{\omega r}{i}} e^{-\frac{3}{4} \lambda \frac{\sin (\Delta \lambda)}{-i \Delta}} \\
=A\left(\cos (\omega r)-\sin (\omega r) e^{\left(\frac{3}{4} \lambda\right) \frac{\sin (\Delta \lambda)}{-i \Delta}}\right.
\end{gathered}
$$

as $\omega \rightarrow 0$, the solution becomes

$$
u=A e^{\left(\frac{3}{4} \lambda\right)} \frac{\sin (\Delta \lambda)}{-i \Delta}
$$

This invariant solution is consistent with one of the solutions obtained by Ibragimov and Gazizov in their paper ([2].)

The plot of this invariant solution is given in Figure (2).

\subsection{Solution 3}

$$
\begin{aligned}
& u=A \omega e^{\frac{\omega r}{i}} e^{-\frac{3}{4} \int_{\psi_{1}}^{\psi_{1}+\omega} d \psi_{1}} \frac{\sin \left(\Delta \int_{\psi_{1}}^{\psi_{1}+\omega} t d \psi_{1}\right)}{-i \Delta \omega} \\
& =A \omega e^{\frac{\omega r}{i}} e^{-\frac{3}{4} \int_{\psi_{1}}^{\psi_{1}+\omega} d \psi_{1}} \frac{d}{d \omega}\left[\frac{\sin \left(\Delta \int_{\psi_{1}}^{\psi_{1}+\omega} t d \psi_{1}\right)}{-i \Delta}\right] \\
& =A \omega e^{\frac{\omega r}{i}} e^{-\frac{3}{4} \int_{\psi_{1}}^{\psi_{1}+\omega} d \psi_{1}} \frac{d \psi_{1}}{d \omega} \frac{d}{d \omega}\left[\frac{\sin \left(\Delta \int_{\psi_{1}}^{\psi_{1}+\omega} t d \psi_{1}\right)}{-i \Delta}\right] \\
& =A \omega e^{\frac{\omega r}{i}} e^{-\frac{3}{4} \int_{\psi_{1}}^{\psi_{1}+\omega} d \psi_{1}} \frac{2 r e^{\frac{2 \omega r}{i}}}{i t}\left[\frac{\cos \left(\Delta \int_{\psi_{1}}^{\psi_{1}+\omega} d \psi_{1}\right)}{-i \Delta}\right] t \\
& =A \omega e^{\frac{\omega r}{i}} e^{-\frac{3}{4} \int_{\psi_{1}}^{\psi_{1}+\omega} d \psi_{1}} \frac{2 r e^{\frac{2 \omega r}{i}}}{i t} t \\
& =A \omega \frac{2 r e^{\frac{3 \omega r}{i}}}{i}
\end{aligned}
$$

\section{But}

$$
\begin{aligned}
& \omega e^{\frac{3 \omega r}{i}}=\omega \cos (3 \omega r)-i \omega \sin (3 \omega r) \\
& \omega^{2} e^{\frac{3 \omega r}{i}}=\omega^{2} \cos (3 \omega r)-i \omega^{2} \sin (3 \omega r) \\
& =\frac{1}{\omega}\left\{\omega^{3} \cos (3 \omega r)-i \omega^{3} \sin (3 \omega r)\right\}
\end{aligned}
$$

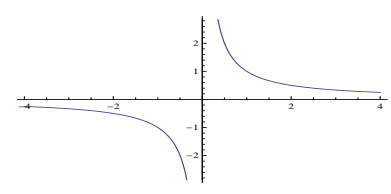

Figure 3: Plot of the solution in (108).

and

$$
\begin{aligned}
& \omega^{3} r^{2} \cos \left(\frac{\omega r}{i}\right)=\omega^{2} e^{3 \omega r} \\
& \omega^{2} e^{\frac{3 \omega r}{i}}=\frac{1}{\omega} \frac{1}{r^{2}} \omega^{2} e^{3 \omega r} \\
& e^{\frac{3 \omega r}{i}}=\frac{1}{\omega} \frac{1}{r^{2}} e^{3 \omega r}
\end{aligned}
$$

We substitute in equation (102) and obtain

$$
u=A \omega \frac{2 r}{i} \frac{1}{\omega} \frac{1}{r^{2}} e^{3 \omega r}
$$

as $\omega \rightarrow 0$, the solution becomes

$$
u=\frac{2 A}{i r}
$$
ure (3).

The plot of this invariant solution is given in Fig-

\section{CONCLUSION}

In this paper, new symmetries were obtained for the Black-Scholes equation, and one was used to determine group invariant solutions. Some of the symmetries are comparable to the ones [2].

\section{APPENDIX A: EULER'S FORMULAS AND THE INFINITESIMAL $\omega$}

It is well-known that Lie's group theoretical methods seek to reduce procedures for solving differential equations of any challenging form to simple ones that may also have the form

$$
a_{0} \ddot{y}+b_{0} \dot{y}+c_{0} y=0,
$$

for $y=y(x)$, with parameters $a_{0}, b_{0}$ and $c_{0}$. It is also that accepted Euler's formulas are suitable for solving such equations. They are:

$$
y=\left\{\begin{array}{cc}
e^{-\frac{b_{0}}{2 a_{0}} x}\left(A e^{-\tilde{\omega} x}+B e^{\tilde{\omega} x}\right), & b_{0}^{2}>4 a_{0} c_{0}, \\
A+B x, & b_{0}^{2}=4 a_{0} c_{0}, \\
e^{-\frac{b_{0}}{2 a_{0}} x}[A \cos (\tilde{\omega} x)] & \\
+B e^{-\frac{b_{0}}{2 a_{0}} x}[\sin (\tilde{\omega} x)], & b_{0}^{2}<4 a_{0} c_{0}
\end{array}\right.
$$


where $\tilde{\omega}=\sqrt{b_{0}^{2}-4 a_{0} c_{0}} /\left(2 a_{0}\right)$.

But there is a problem with this system: It does not reduce to $y=A+B x$ when $b_{0}=c_{0}=0$. This is because Euler did not solve the equation to get the formulas. There has never been a need to do so, primarily because the formulas have been very successful in applications, and they still are.

The need for an exact solution here, is driven by the desire understand solutions for equation (9) through symmetry methods. It is impossible through Euler's formulas. To get such exact formula, first let

$$
y=\beta z,
$$

with $\beta=\beta(x)$ and $z=z(x)$, so that

$$
\dot{y}=\dot{\beta} z+\beta \dot{z},
$$

and

$$
\ddot{y}=\ddot{\beta} z+2 \dot{\beta} \dot{z}+\beta \ddot{z} .
$$

These transform (109) into

$a_{0}(\ddot{\beta} z+2 \dot{\beta} \dot{z}+\beta \ddot{z})+b_{0}(\dot{\beta} z+\beta \dot{z})+c_{0} \beta z=0$.

That is,

$a_{0} \beta \ddot{z}+\left(2 a_{0} \dot{\beta}+b_{0} \beta\right) \dot{z}+\left(a_{0} \ddot{\beta}+b_{0} \dot{\beta}+c_{0} \beta\right) z=0$

Choosing $\beta$ to satisfy $2 a_{0} \dot{\beta}+b_{0} \beta=0$ simplifies equation (111). That is,

$$
\beta=C_{00} e^{\frac{-b_{0}}{2 a_{0}} x},
$$

for some constant $C_{00}$. Equation (111) assumes the form

$$
\ddot{z}=-\frac{a_{0} \ddot{\beta}+b_{0} \dot{\beta}+c_{0} \beta}{a_{0} \beta} z .
$$

That is,

$$
\ddot{z}=\left(\frac{b_{0}^{2}-4 a_{0} c_{0}}{4 a_{0}^{2}}\right) z .
$$

But $\ddot{z}$ can be written as $\dot{z} d z / d x$. Therefore,

$$
\dot{z} \frac{d \dot{z}}{d z}=\left(\frac{b_{0}^{2}-4 a_{0} c_{0}}{4 a_{0}^{2}}\right) z,
$$

or

$$
\dot{z} d \dot{z}=\left(\frac{b_{0}^{2}-4 a_{0} c_{0}}{4 a_{0}^{2}}\right) z d z .
$$

That is,

$$
\frac{\dot{z}^{2}}{2}=\left(\frac{b_{0}^{2}-4 a_{0} c_{0}}{4 a_{0}^{2}}\right) \frac{z^{2}}{2}+C_{01}
$$

for some constant $C_{01}$. That is,

$$
\dot{z}=\sqrt{\left(\frac{b_{0}^{2}-4 a_{0} c_{0}}{4 a_{0}^{2}}\right) \frac{z^{2}}{2}+C_{01}},
$$

or

$$
\frac{d z}{\sqrt{\left(\frac{b_{0}^{2}-4 a_{0} c_{0}}{4 a_{0}^{2}}\right) z^{2}+2 C_{01}}}=d x
$$

That is,

$$
\frac{d z}{\sqrt{A_{00}^{2}-z^{2}}}=\sqrt{-\frac{b_{0}^{2}-4 a_{0} c_{0}}{4 a_{0}^{2}}} d x
$$

with $A_{00}^{2}=2 C_{01} / \sqrt{-\frac{b_{0}^{2}-4 a_{0} c_{0}}{4 a_{0}^{2}}}$. Hence,

$$
z=\frac{2 C_{01}}{\sqrt{-\frac{b_{0}^{2}-4 a_{0} c_{0}}{4 a_{0}^{2}}}} \sin \left(\sqrt{-\frac{b_{0}^{2}-4 a_{0} c_{0}}{4 a_{0}^{2}}} x+C_{02}\right),
$$

for some constant $C_{02}$. That is,

$y=C_{00} e^{\frac{-b_{0}}{2 a_{0}} x} \frac{2 C_{01}}{\sqrt{-\frac{b_{0}^{2}-4 a_{0} c_{0}}{4 a_{0}^{2}}}} \sin \left(\sqrt{-\frac{b_{0}^{2}-4 a_{0} c_{0}}{4 a_{0}^{2}}} x+C_{02}\right)$.

Letting

$$
\bar{\omega}=\sqrt{-\frac{b_{0}^{2}-4 a_{0} c_{0}}{4 a_{0}^{2}}}
$$

we have

$$
\begin{aligned}
& y=C_{00} e^{\frac{-b_{0}}{2 a_{0}} x} \frac{2 C_{01}}{\bar{\omega}} \sin \left(\bar{\omega} x+C_{02}\right), \\
& \text { or } \\
& y=C_{00} e^{\frac{-b_{0}}{2 a_{0}} x} \quad 2 C_{01} \quad\left[\frac{\sin \left(C_{02}\right)}{\bar{\omega}} \cos (\bar{\omega} x)+\right.
\end{aligned}
$$$$
\left.\cos \left(C_{02}\right) \frac{\sin (\bar{\omega} x)}{\bar{\omega}}\right] \text {. }
$$

A reduction to the trivial case $\ddot{y}=0$ requires that $\sin \left(C_{02}\right)=C_{03} \sin (\bar{\omega})$ and $\cos \left(C_{02}\right)=C_{04} \cos (\bar{\omega})$. That is, $C_{03}^{2}+C_{04}^{2}=1$. Hence,

$y=C_{00} e^{\frac{-b_{0}}{2 a_{0}} x} \quad 2 C_{01} \quad\left[\frac{C_{03} \sin (\bar{\omega})}{\bar{\omega}} \cos (\bar{\omega} x)+\right.$ $\left.C_{04} \cos (\bar{\omega}) \frac{\sin (\bar{\omega} x)}{\bar{\omega}}\right]$, or simply

$$
\begin{array}{cc}
y & = \\
C_{00} e^{\frac{-b_{0}}{2 a_{0}} x} 2 C_{01} \frac{C_{03} \sin (\bar{\omega}) \cos (\bar{\omega} x)}{\bar{\omega}} \\
+C_{00} e^{\frac{-b_{0}}{2 a_{0}} x} 2 C_{01} \frac{C_{04} \sin (\bar{\omega} x)}{\bar{\omega}} .
\end{array}
$$

It is very vital to indicate that if the parameters $\bar{\omega}$ in the denominator and $\sin (\bar{\omega})$ are absorbed into the coefficients $C_{01}$ and $C_{03}$, then formula (112) would reduce to one of Euler's formulas. But the consequences would be fatal, as formula (112) would not reduce to $y=A+B x$ when $b_{0}=c_{0}=0$, that is, when $\bar{\omega}=0$.

Unfortunately, this result cannot be found in any university textbook. 


\section{APPENDIX B: Useful limit results}

It is true that

$$
\lim _{\mu \rightarrow 0}\left\{\frac{\sin \left(\frac{\mu t}{i}\right)}{\mu}\right\}=\frac{t}{i} .
$$

This can be written in the form

$$
\lim _{\mu \rightarrow 0}\left\{\frac{\sin \left(\frac{\mu x}{i}\right)}{\mu}-\frac{t}{i}\right\}=0
$$

or

$$
\lim _{\mu \rightarrow 0}\left\{\frac{\sin \left(\frac{\mu t}{i}\right)}{\mu}-\frac{t}{i} \cos \left(\frac{\mu t}{i}\right)\right\}=0 .
$$

Removing the 'lim' for greater clarity:

$$
\frac{\sin \left(\frac{\mu t}{i}\right)}{\mu}=\frac{t}{i} \cos \left(\frac{\mu t}{i}\right) .
$$

That is,

$$
\sin \left(\frac{\mu t}{i}\right)=\frac{t}{i} \mu \cos \left(\frac{\mu t}{i}\right)
$$

or

$$
\cos \left(\frac{\mu t}{i}\right)=\frac{i \sin \left(\frac{\mu t}{i}\right)}{t} .
$$

We then have

$$
\frac{\cos \left(\frac{\mu t}{i}\right)}{\mu^{q}}=\mu \frac{\cos \left(\frac{\mu t}{i}\right)}{\mu^{q+1}} .
$$

Carrying out the derivative on the right hand side:

$$
\frac{\cos \left(\frac{\mu t}{i}\right)}{\mu^{q}}=\frac{-\mu\left(\frac{t}{i}\right) \sin \left(\frac{\mu t}{i}\right)+\cos \left(\frac{\mu t}{i}\right)}{\mu^{q+1}} .
$$

Substituting (114):

$$
\frac{\cos \left(\frac{\mu t}{i}\right)}{\mu^{q}}=\frac{-\mu^{2}\left(\frac{t}{i}\right)^{2} \cos \left(\frac{\mu t}{i}\right)+\cos \left(\frac{\mu t}{i}\right)}{\mu^{q+1}} .
$$

That is,

$$
\mu \cos \left(\frac{\mu t}{i}\right)=\mu^{2} t^{2} \cos \left(\frac{\mu t}{i}\right)+\cos \left(\frac{\mu t}{i}\right),
$$

which can be expressed in the form

$$
\mu^{2} \cos \left(\frac{\mu t}{i}\right)-\mu^{3} t^{2} \cos \left(\frac{\mu t}{i}\right)=\frac{i}{t} \sin \left(\frac{\mu t}{i}\right) .
$$

Since $\sin \left(\frac{\mu t}{i}\right)=0$ for $\mu$ small, it follows then that

$$
\mu^{2} \cos \left(\frac{\mu t}{i}\right)=\mu^{3} t^{2} \cos \left(\frac{\mu t}{i}\right) .
$$

Since $e^{\mu t}$ ca be expressed in the form $\cos (\mu t / i)+$ $i \sin (\mu t / i)$, then

$$
\mu^{2} e^{\mu t}=\mu^{3} t^{2} \cos \left(\frac{\mu t}{i}\right),
$$

so that

$$
\sqrt{\mu} e^{\mu t / 4}=\left[\mu^{3} \cos \left(\frac{\mu t}{i}\right)\right]^{\frac{1}{4}} \sqrt{t},
$$

or

$$
\sqrt{\mu} e^{-\mu t / 4}=\left[\mu^{3} \cos \left(\frac{\mu t}{i}\right)\right]^{\frac{1}{4}} \sqrt{t},
$$

Therefore (119) and (123) can then be written in the form

$$
u=\frac{\sqrt{\mu}}{\left[\mu^{3} \cos \left(\frac{\mu t}{i}\right)\right]^{\frac{1}{4}} \sqrt{t}} \phi(\eta),
$$

with $\mu=\omega^{4}\left(\omega^{2}-1\right)$ in the case of (119) and $\mu=$ $\omega^{4}\left(\omega^{2}+1\right)$ for $(123)$. That is,

$$
u=\frac{1}{\sqrt{\left(\omega^{2}-1\right) t} \omega^{2}} \phi(\eta)
$$

for (119), and

$$
u=\frac{1}{\sqrt{\left(\omega^{2}+1\right) t} \omega^{2}} \phi(\eta)
$$

for (121). 
References:

[1] Blumen,G.W. Anco,S.C. 2002.

Symmetries and Integration methods for

Differential Equations. New York. Springer-

Verlag.

[2] Gazizov, R.K and Ibragimov, N.H.1998.

Lie Symmetry Analysis of Differential

Equations in Finance. Nonlinear Dynamics

17:387 - 407. June.

[3] Ibragimov, N.H.1999. Elementary Lie Group Analysis and Ordinary Differential Equations. London. J. Wiley \& Sons Ltd.

[4] Miller, R.M. 1993. Option Valuation.

Economic and Financial Modelling with

Mathematica. Springer Verlag.
[5] Silberberg,G.(2001).Derivative Pricing with Symmetry

Analysis.http://www.econ.ceu.hu/download/th esis/Thesis-Silberberg. pdf

[6] Ivan V. Kazachkov, Numerical Simulation of the Turbulent Two-Phase Jet

Pages 107-119, Volume 2, 2020, Engineering World

[7] Kittipong Tripetch, An Analysis of an Input Impedance of a Regulated Cascode Cross Couple Power Amplifier, Pages 100106, Volume 2, 2020, Engineering World

[8] Nazli Goker, Mehtap Dursun, Elif Dogu, A Cognitive Mapping Approach for Evaluation of Success Indicators of IT Transformation Project, Pages 96-99, , Volume 2, 2020, Engineering World 
Sources of funding for research presented in a scientific article or scientific article itself

Report potential sources of funding if there is any

We thank the Reviewer for their positive comment and careful review, which helped improve the manuscript. 


\section{Creative Commons Attribution License 4.0} (Attribution 4.0 International, CC BY 4.0)

This article is published under the terms of the Creative Commons Attribution License 4.0

https://creativecommons.org/licenses/by/4.0/deed.en US 